\title{
On the Interplay between Interval Dimension and Dimension *
}

\author{
S. Felsner $^{\dagger} \quad$ M. Habib $\stackrel{\text { R.H. Möhring }}{\S}$
}

\section{Abstract}

This paper investigates a transformation $P \rightarrow Q$ between partial orders $P, Q$ that transforms the interval dimension of $P$ to the dimension of $Q$, i.e., $\operatorname{idim}(P)=$ $\operatorname{dim}(Q)$. Such a construction has been shown before in the context of Ferrer's dimension by Cogis [2]. Our construction can be shown to be equivalent to his, but it has the advantage of (1) being purely order-theoretic, (2) providing a geometric interpretation of interval dimension similar to that of Ore [15] for dimension, and (3) revealing several somewhat surprising connections to other order-theoretic results.

For instance, the transformation $P \rightarrow Q$ can be seen as almost an inverse of the well-known split operation, it provides a theoretical background for the influence of edge subdivision on dimension (e.g., the results of Spinrad [17]) and interval dimension, and it turns out to be invariant with respect to changes of $P$ that do not alter its comparability graph, thus providing also a simple new proof for the comparability invariance of interval dimension.

\footnotetext{
${ }^{*}$ This work was partially supported by the DFG and by the DAAD Project PROCOPE.

†TU-Berlin, Berlin, Germany

$\ddagger$ L.R.I.M.M., Montpellier, France

$\S \mathrm{TU}-$ Berlin, Berlin, Germany
} 


\section{Introduction}

An extension $Q$ of a partial order $P$ is an order on the same elements that contains all the ordered pairs of $P$, i.e., $x<y$ in $P$ implies $x<y$ in $Q$. A family $\left\{Q_{1}, \ldots, Q_{k}\right\}$ of extensions of $P$ is said to realize $P$ or to be a realizer of $P$ iff $P=Q_{1} \cap \ldots \cap Q_{k}$, i.e., $x<y$ in $P$ iff $x<y$ in $Q_{i}$ for each $i, 1 \leq i \leq k$. If we restrict the $Q_{i}$ to belong to a special class of orders and seek for a minimum size realizer we come up with a concept of dimension with respect to the special class.

A linear extension of $P$ is an extension which is a chain. Dushnik and Miller [5] defined the dimension of a partial order $P$, denoted $\operatorname{dim}(P)$, as the smallest integer $k$ for which there exist $k$ linear extensions realizing $P$. Let the realizer $\left\{L_{1}, \ldots, L_{k}\right\}$ of an order $P$ with $|P|=n$ be made by the linear extensions $L_{i}=\left(x_{i 1}<x_{i 2}<\ldots<x_{i n}\right)$. With every $x \in P$ we then associate the vector $\left(x^{1}, \ldots, x^{k}\right) \in \mathbf{R}^{k}$, where $x^{i}$ gives the position (coordinate) of $x$ in $L_{i}$. This mapping of the points of $P$ to points of $\mathbf{R}^{k}$ embeds $P$ into the componentwise ordering of $\mathbf{R}^{k}$. Ore [15] defined $\operatorname{dim}(P)$ as the minimum $k$ such that $P$ embeds into $\mathbf{R}^{k}$ in this way. The projections of such an embedding on each coordinate yield a realizer of $P$. The extensions of this realizer need not be linear extensions of $P$, but it is straightforward to transform them into linear extensions. Therefore, Ore dimension and Dushnik-Miller dimension are equivalent concepts.

A partial order $P$ is an interval order if it can be represented by assigning to each element $x \in P$ an open interval $I_{x}=\left(a_{x}, b_{x}\right)$ of the real line, such that $x<y$ in $P$ iff $b_{x} \leq a_{y}$. Such a collection of intervals is called an interval representation of $P$. Fishburn [7] characterized interval orders as those orders that do not contain a 4-point subset forming two disjoint 2 -chains, i.e., that contain no $\mathbf{2}+\mathbf{2}$. Interval dimension, denoted $\operatorname{idim}(P)$, is defined by using interval extensions instead of linear extensions. Since linear orders are interval orders we obtain the trivial inequality

$$
\operatorname{idim}(P) \leq \operatorname{dim}(P)
$$

It is well known that interval orders of large dimension exist (see $[16,1,9]$ ). Hence, the gap between $\operatorname{idim}(P)$ and $\operatorname{dim}(P)$ may be arbitrarily large.

Let $\mathcal{I}=\left\{I_{1}, \ldots, I_{k}\right\}$ be an interval realizer of $P$ and fix an open interval 
representation of each interval order $I_{j}$. Let $\left(a_{x}^{j}, b_{x}^{j}\right)$ be the interval corresponding to $x \in P$ in the representation of $I_{j}$. We now define a box embedding of $P$ to $\mathbf{R}^{k}$. With $x \in P$ we associate the box $\prod_{j}\left(a_{x}^{j}, b_{x}^{j}\right) \subseteq \mathbf{R}^{k}$. Each of these boxes is uniquely determined by its upper extreme corner $u_{x}=\left(b_{x}^{1}, \ldots, b_{x}^{k}\right)$ and its lower extreme corner $l_{x}=\left(a_{x}^{1}, \ldots, a_{x}^{k}\right)$. Obviously $x<y$ in $P$ iff $u_{x} \leq l_{y}$ componentwise. The projections of a box embedding onto each coordinate yield an interval realizer, so the concepts of box embeddings and interval realizers are equivalent. For interval dimension the box embeddings thus play the role of the above-mentioned point embeddings into $\mathbf{R}^{k}$ introduced by Ore for dimension.

A box embedding depends not only on the realizer $\mathcal{I}$ of $P$, but also on the representations of the $I_{j}$. Now we define the partial order $\mathcal{B}(\mathcal{I})$ of extreme corners associated with a box embedding or, equivalently, with an interval realizer $\mathcal{I}$ of $P$. The vertices of $\mathcal{B}(\mathcal{I})$ are the at most $2 n$ different lower resp. upper extreme corners of elements of $P$. The order relation of $\mathcal{B}(\mathcal{I})$ is given by the componentwise order in $\mathbf{R}^{k}$.

By definition we have an embedding of $\mathcal{B}(\mathcal{I})$ in $\mathbf{R}^{k}$, so $\operatorname{dim} \mathcal{B}(\mathcal{I}) \leq k=$ $\operatorname{idim}(P)$. The starting point of our investigations was the following question concerning the interplay between dimension and interval dimension:

$$
\text { Is } \operatorname{dim} \mathcal{B}(\mathcal{I})=\operatorname{idim}(P) \text { ? }
$$

In Section 2 we define a transformation $P \rightarrow B(P)$ such that $\operatorname{idim}(P)=$ $\operatorname{dim} B(P)$. We provide two interpetations of this transformation, a combinatorial one and a geometrical one. In the combinatorial interpretation the elements of $B(P)$ are subsets of $P$. In the geometrical interpretation $B(P)$ is the poset $\mathcal{B}\left(\mathcal{I}^{*}\right)$ of extreme corners of $\mathcal{I}^{*}$. Here $\mathcal{I}^{*}$ is a box embedding obtained from an arbitrary box embedding $\mathcal{I}$ by a normalizing procedure. From the proofs we obtain an affirmative answer to the question above.

Section 3 investigates several consequences to and relations with other ordertheoretic results. First, we study the transformation $P \rightarrow B(P)$ on special partial orders of height 1 . In particular we show that the standard example $S_{n}$ of a $n$ dimensional order is an (almost) fixed point of the transformation $P \rightarrow B(P)$. Therefore, $\operatorname{dim}\left(S_{n}\right)=\operatorname{idim}\left(S_{n}\right)$.

Second, we investigate the relationship with the split operation. This has a surprising consequence for the iterated transformation $P \rightarrow B(P) \rightarrow B^{2}(P) \rightarrow$ 
$\ldots B^{k}(P) \rightarrow \ldots$ For every $n$ there are partial orders $P$ such that $0 \leq \operatorname{dim}(P)-$ $\operatorname{dim} B^{k}(P) \leq 2$ for all $k \leq n$ but $\operatorname{dim}(P)-\operatorname{dim} B^{n+1}(P) \geq m$, where $m$ is arbitrary.

Third, we relate the interval dimension of subdivisions of $P$ to the dimension of $P$, thus providing a theoretical framework for the examples of Spinrad [17].

Finally, we show the comparability invariance of the transformation $P \rightarrow$ $B(P)$, which, as a consequence, gives another proof that the interval dimension is a comparability invariant. Some remarks concerning the recognition-complexity of special classes of orders and graphs close the paper.

\section{The main result}

In the last section we defined the partial order $\mathcal{B}(\mathcal{I})$ of extreme corners associated with a box embedding of $P$ in $\mathbf{R}^{k}$. With the next lemmas we show that $\mathcal{B}(\mathcal{I})$ inherits some structure which is independent of the realizer $\mathcal{I}$ leading to the box embedding.

Lemma 1 Let $\mathcal{B}(\mathcal{I})$ be the partial order of extreme corners of a box representation of $P$.

a) If the lower extreme corners of $x$ and $y$ are comparable in $\mathcal{B}(\mathcal{I})$, e.g., $l_{x} \leq$ $l_{y}$, then the predecessor sets of $x$ and $y$ in $P$ are ordered by inclusion, i.e., $\operatorname{Pred}_{P}(x) \subseteq \operatorname{Pred}_{P}(y)$.

b) If the upper extreme corners of $x$ and $y$ are comparable in $\mathcal{B}(\mathcal{I})$, e.g., $u_{x} \leq u_{y}$, then the successor sets of $x$ and $y$ in $P$ are ordered by (reversed) inclusion, i.e., $\operatorname{Succ}_{P}(x) \supseteq \operatorname{Succ}_{P}(y)$.

c) If the lower extreme corner of $x$ and the upper extreme corner of $y$ are related by $l_{x} \leq u_{y}$ then $\operatorname{Pred}_{P}(z) \supseteq \operatorname{Pred}_{P}(x)$ for all $z \in \operatorname{Succ}_{P}(x)$ or, equivalently, $\operatorname{Pred}_{P}(x) \subseteq \bigcap \operatorname{Pred}_{P}(z)$.

$$
z \in \operatorname{Succ}_{P}(y)
$$

d) If $u_{x} \leq l_{y}$ then $\bigcap \operatorname{Pred}_{P}(z) \subseteq \operatorname{Pred}_{P}(y)$. $z \in \operatorname{Succ}_{P}(x)$

Proof. a) From $l_{x} \leq l_{y}$ we obtain $a_{x}^{j} \leq a_{y}^{j}$ for all $j$. Therefore, in each $I_{j}, x$ has less predecessors than $y$, i.e., $\operatorname{Pred}_{j}(x) \subseteq \operatorname{Pred}_{j}(y)$. The claim now follows from 
$\operatorname{Pred}_{P}(x)=\bigcap_{j} \operatorname{Pred}_{j}(x)$ since the $I_{j}$ realize $P$.

b) The proof of this part is symmetric to part a.

c) From $l_{x} \leq u_{y}$ we have $a_{x}^{j} \leq b_{y}^{j}$. If $z \in \operatorname{Succ}(y)$, then necessarily $a_{z}^{j} \geq b_{y}^{j} \geq a_{x}^{j}$. Hence, $\operatorname{Pred}_{j}(z) \supseteq \operatorname{Pred}_{j}(x)$ for all $j$. The claim follows.

d) From $u_{x} \leq l_{y}$ we immediately obtain $x \leq y$, i.e., $y \in \operatorname{Succ}(x)$, therefore, $\operatorname{Pred}(y) \supseteq \bigcap \operatorname{Pred}(z)$.

$z \in \operatorname{Succ}(x)$

All statments except the conclusion part of $\mathrm{b}$ use only the sets $\operatorname{Pred}_{P}(x)$ and

$\bigcap \operatorname{Pred}_{P}(z)$. This irregularity is resolved with the next lemma. $z \in \operatorname{Succ}_{P}(x)$

Lemma $2 \bigcap_{z \in \operatorname{Succ}(x)} \operatorname{Pred}(z) \supseteq \bigcap_{z \in \operatorname{Succ}(y)} \operatorname{Pred}(z) \quad$ if and only if $\quad \operatorname{Succ}(x) \subseteq \operatorname{Succ}(y)$.

Proof. The 'if' direction is trivial. We now prove the 'only if' direction. Let $z \in \operatorname{Succ}(x)$ and note that $y \in \bigcap_{z \in \operatorname{Succ}(y)} \operatorname{Pred}(z)$. From the assumed inclusion we obtain $y \in \operatorname{Pred}(z)$, hence $z \in \operatorname{Succ}(y)$

Definition 1 With each vertex $x$ of a partial order $P$ we associate the lower set $L(x)=\operatorname{Pred}_{P}(x)$ and the upper set $U(x)=\bigcap \operatorname{Pred}_{P}(z)$. The case $x \in$ $\operatorname{Max}(P)$ is settled by the convention $U(x)=P$. $z \in \operatorname{Succ}_{P}(x)$

Define $B(P)=\{L(x), U(x): x \in P\}$ ordered by setinclusion.

Note that this construction is in fact equivalent with Cogis' construction in the context of Ferrers dimension [2]. Cogis also uses $L(x)$, but replaces $U(x)$ by the equivalent set $\{z \in P: \operatorname{Succ}(x) \subseteq \operatorname{Succ}(z)\}$. He also proves Theorem 2, but in a different way and without the geometrical interpretation that our approach is based on.

The preceding lemmas prove that $l_{x} \rightarrow L(x)$ and $u_{x} \rightarrow U(x)$ together form an order preserving mapping from $B(\mathcal{I})$ to $B(P)$, hence

$$
\operatorname{idim}(P) \geq \operatorname{dim} \mathcal{B}(\mathcal{I}) \geq \operatorname{dim} B(P)
$$

To get more structure into interval realizers we now introduce a procedure that transforms a interval extension $I=\left\{\left(a_{x}, b_{x}\right): x \in P\right\}$ of $P$ into its $P$ normalization $I^{*}=\left\{\left(a_{x}^{*}, b_{x}^{*}\right): x \in P\right\}$. 
- In the first step of the $P$-normalization we update left endpoints:

$a_{x}^{*}=\max \left\{b_{z}: z \in \operatorname{Pred}(x)\right\}$ if $x$ is not minimal,

$a_{x}^{*}=\min \left\{a_{z}: z \in \operatorname{Min}(P)\right\}$ if $x$ is minimal.

- In the second step we update right endpoints:

$b_{x}^{*}=\min \left\{a_{z}^{*}: z \in \operatorname{Succ}(x)\right\}$ if $x$ is not maximal,

$b_{x}^{*}=\max \left\{b_{z}: z \in \operatorname{Max}(P)\right\}$ if $x$ is maximal.

Note that the interval order $I^{*}$ need not be isomorphic to $I$. In general $I^{*}$ is a suborder of $I$ and a minimal interval extension of $P$ if all the $a_{x}, b_{x}$ were different.

If $P$ is realized by $\mathcal{I}=\left\{I_{1}, \ldots, I_{k}\right\}$ then $\mathcal{I}^{*}=\left\{I_{1}^{*}, \ldots, I_{k}^{*}\right\}$ realize $P$ as well. We call the box embedding corresponding to $\mathcal{I}^{*}$ the normalized box embedding of $\mathcal{I}$. For an example see Figure 1.
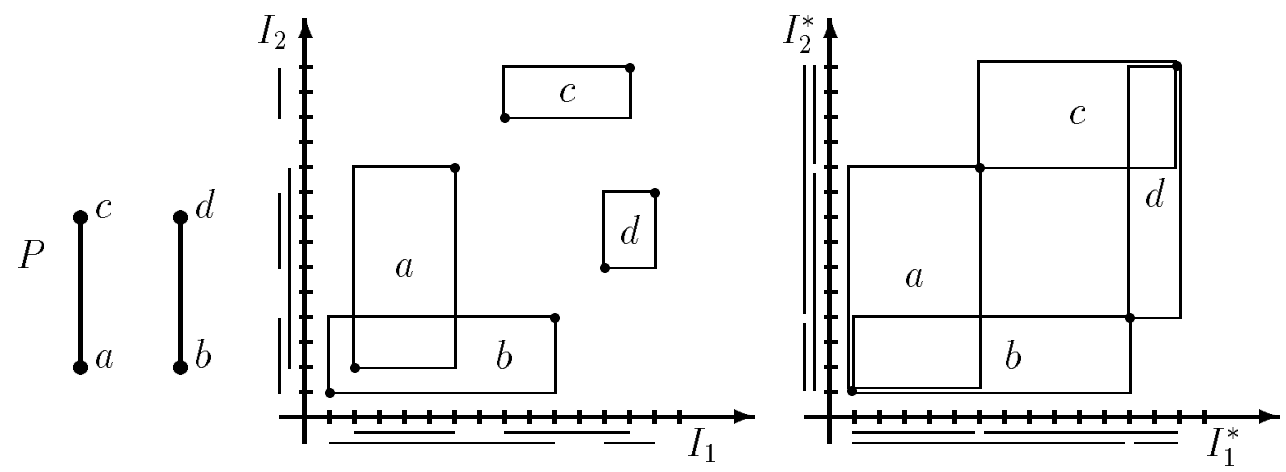

Figure 1: $P$, an interval realizer of $P$ and its normalization

After normalizing we have a realizer $\mathcal{I}^{*}=\left\{I_{1}^{*}, \ldots, I_{k}^{*}\right\}$ of $P$, interval representations $\left(a_{x}^{j *}, b_{x}^{j *}\right)$ and an associated partial order of extreme corners $\mathcal{B}\left(\mathcal{I}^{*}\right)=$ $\left\{l_{x}^{*}, u_{x}^{*}: x \in P\right\}$. The next theorem shows that the geometrically defined $\mathcal{B}\left(\mathcal{I}^{*}\right)$ and the combinatorially defined $B(P)$ are isomorphic.

Theorem 1 If $\mathcal{I}^{*}$ is a normalized realizer of $P$ then $\mathcal{B}\left(\mathcal{I}^{*}\right)=B(P)$.

Proof. First observe that both partial orders have a least element generated by $x \in \operatorname{Min}(P)$ as $l_{x}^{*}$ and $L(x)$, respectively, and a greatest element generated by $x \in \operatorname{Max}(P)$ as $u_{x}^{*}$ and $U(x)$, respectively. 
Moreover, $l_{x}^{*} \rightarrow L(x)$ and $u_{x}^{*} \rightarrow U(x)$ defines an order preserving mapping by the remarks preceeding (2). To show the converse we distinguish four cases:

$U(x) \subseteq L(y)$. We know that $x \in U(x)$, so $x \in \operatorname{Pred}(y)$. Since $I_{j}^{*}$ is an interval extension of $P$, we obtain $b_{x}^{j *} \leq a_{y}^{j *}$ for all $j$. Hence $u_{x}^{*} \leq l_{y}^{*}$.

$L(x) \subseteq L(y)$. Remember that $a_{x}^{j *}=\max \left\{b_{z}^{j}: z \in \operatorname{Pred}(x)\right\}$ and $a_{y}^{j *}=$ $\max \left\{b_{z}^{j}: z \in \operatorname{Pred}(y)\right\}$. By assumption $\operatorname{Pred}(x) \subseteq \operatorname{Pred}(y)$, so $a_{x}^{j *} \leq a_{y}^{j *}$ and $l_{x}^{*} \leq l_{y}^{*}$.

$U(x) \subseteq U(y)$. By Lemma 2, this is equivalent to $\operatorname{Succ}(x) \supseteq \operatorname{Succ}(y)$. Now $u_{x}^{*} \leq u_{y}^{*}$ follows symmetrically with the second case.

$L(x) \subseteq U(y)$. Since $I_{j}^{*}$ is normalized, there are $z_{0} \in \operatorname{Pred}(x)$ and $z_{1} \in \operatorname{Succ}(y)$ with $a_{x}^{j *}=b_{z_{0}}^{j}$ and $b_{y}^{j *}=a_{z_{1}}^{j *}$. The hypothesis provides $z_{0} \leq z_{1}$, hence $a_{x}^{j *}=b_{z_{0}}^{j} \leq$ $b_{z_{0}}^{j *} \leq a_{z_{1}}^{j *}=b_{y}^{j *}$. The validity of this inequality for all $j$ again gives $l_{x}^{*} \leq u_{y}^{*}$.

We are ready now, to prove our main theorem about interval dimension and dimension.

Theorem $2 \operatorname{dim} B(P)=\operatorname{idim}(P)$.

Proof. As Inequality (2) we allready have obtained $\operatorname{dim} B(P) \leq i \operatorname{dim}(P)$. For the converse we need two arguments. We first show that a linear extension $L$ of $B(P)$ induces an interval extension $I_{L}$ of $P$. Secondly, we prove that if $L_{1}, \ldots, L_{k}$ is a realizer of $B(P)$, then the induced interval extensions $I_{L_{1}}, \ldots, I_{L_{k}}$ form an interval realizer of $P$.

Let $L=M_{1}, M_{2}, \ldots, M_{r}$ be a linear extension of $B(P)$. For each $x \in P$ there are $i, j \in\{1, \ldots, r\}$ such that $M_{i}=L(x)$ and $M_{j}=U(x)$. From $L(x) \subseteq U(x)$ and $x \notin L(x), x \in U(x)$ we obtain that $i<j$. So we can associate with $x$ a unique interval $\left(a_{x}, b_{x}\right)$ which is defined to be $(i, j)$. We now show that the interval order $I_{L}$ induced by the interval representation $\left\{\left(a_{x}, b_{x}\right): x \in P\right\}$ is an extension of $P$. If $x<y$ in $P$, then $U(x) \subseteq L(y)$, and thus, with $M_{i}=U(x)$ and $M_{j}=L(y)$, $b_{x}=i \leq j=a_{y}$, which implies $x<y$ in $I_{L}$.

Let $\left\{L_{1}, \ldots, L_{k}\right\}$ be a realizer for $B(P)$ and let $I_{j}=I_{L_{j}}$, for $1 \leq j \leq k$. The family $\left\{I_{1}, \ldots, I_{k}\right\}$ of interval extensions of $P$ is an interval realizer iff all incomparabilities $x \| y$ of $P$ are realized. If $x \| y$ in $P$, then $U(x) \nsubseteq L(y)$ since $x \in U(x)$ but $x \notin L(y)$. Therefore, $L(y)$ precedes $U(x)$ in some $L_{j}$, which gives 
$a_{y}^{j}<b_{x}^{j}$. The symmetric argument yields an $i$ with $a_{x}^{i}<b_{y}^{i}$. Both inequalities together give $x \| y$ in $\bigcap_{j} I_{j}$.

This theorem together with Inequality $(2)$ shows that $\operatorname{dim} \mathcal{B}(\mathcal{I})$ is independent of the interval realizer $\mathcal{I}$.

\section{Consequences}

In the previous section we introduced the operation $P \rightarrow B(P)$ mapping partial orders to partial orders. We will now investigate several connections to other order-theoretical topics and results. Note that $B(P)$ always has a greatest and a least element. We adopt the convention of calling orders with this property bounded and denote by $Q \rightarrow \widehat{Q}$ the bounding of a partial order $Q$, i.e., $\hat{Q}$ is the order resulting from $Q$ by adjoining a new greatest and a new least element.

We first look at the effect of the operator $B$ applied to special classes of orders.

\subsection{Transformation rules for special posets}

Let $S_{n}$ denote the standard poset of dimension $n$, i.e., the set of all 1-element and $(n-1)$-subsets of an $n$ element set ordered by setinclusion. Then

$$
B\left(S_{n}\right)=\widehat{S_{n}}
$$

Let $C_{r}$ denote the $r$-cycle. The $r$-cycle is the 3 -dimensional poset on $2 r$ elements $\left\{x_{1}, y_{1}, x_{2}, y_{2}, \ldots, x_{r}, y_{r}\right\}$ with comparabilities.

$$
\begin{gathered}
x_{1}<y_{1}, y_{1}>x_{2}, x_{2}<y_{2}, \ldots x_{r}<y_{r}, y_{r}>x_{1} \\
B\left(C_{r}\right)=\widehat{C_{r}}
\end{gathered}
$$

Let the Hasse diagram of $T$ be a tree. The truncation of $T$, denoted by $\operatorname{tr}(T)$, is the induced tree on the non-leaf vertices of $T$. Then

$$
B(T)=\widehat{t r(T)}
$$

In particular (3) shows that the standard example $S_{n}$ of a $n$-dimensional order is (up to closures) a fixed point of the operation $P \rightarrow B(P)$, thus showing again that, for every $n \geq 3$, there is are orders $P$ with $\operatorname{dim}(P)=\operatorname{idim}(P)=n$. 


\section{2 $\quad B$ as an inverse of the split operation}

We now turn to the natural question, whether, for every bounded partial order $Q$ there is some $P$ with $Q=B(P)$. The next theorem answers this question affirmatively. Moreover, it turns out that the operation $P \rightarrow B(P)$ is an almost left inverse of the split operation $S$ which has applications in different branches of poset theory (see, e.g., $[21,8,6]$ ). The split $S[P]$ of a partial order $P$ is the order of height one with minimal elements $\left\{x^{\prime}: x \in P\right\}$ maximal elements $\left\{x^{\prime \prime}: x \in P\right\}$ and ordered pairs $x^{\prime}<y^{\prime \prime}$ iff $x \leq y$ in $P$.

Theorem $3 B(S[P])=\widehat{P}$.

Proof. For $x \in P$ let Pred $[x]=\operatorname{Pred}(x) \cup\{x\}$. Obviously, $P$ is isomorphic to the setsystem $\{\operatorname{Pred}[x]: x \in P\}$ ordered by inclusion. We will show that the elements of $B(S[P])$ are just the 'primed' sets Pred $[x]$, i.e., (Pred $[x])^{\prime}=\left\{x^{\prime}: x \in \operatorname{Pred}[x]\right\}$, together with the greatest element $\left\{x^{\prime}, x^{\prime \prime}: x \in P\right\}$ and the least element $\emptyset$. We have

$$
\begin{aligned}
& L\left(x^{\prime}\right)=\emptyset, \text { and } \\
& U\left(x^{\prime}\right)=\bigcap_{z^{\prime \prime} \in \operatorname{Succ}\left(x^{\prime}\right)} \operatorname{Pred}\left(z^{\prime \prime}\right)=\bigcap_{z \in \operatorname{Succ}[x]}(\operatorname{Pred}[z])^{\prime} . \text { Since } x \in \operatorname{Succ}[x], U\left(x^{\prime}\right) \subseteq(\operatorname{Pred}[x])^{\prime} .
\end{aligned}
$$

On the other hand Pred $[x] \subset \operatorname{Pred}[z]$ for $z \in \operatorname{Succ}(x)$. Together, this gives $U\left(x^{\prime}\right)=(\operatorname{Pred}[x])^{\prime}$. Similarly, we obtain

$$
\begin{aligned}
& L\left(x^{\prime \prime}\right)=\operatorname{Pred}\left(x^{\prime \prime}\right)=(\operatorname{Pred}[x])^{\prime} . \text { Finally, } \\
& U\left(x^{\prime \prime}\right)=\left\{x^{\prime}, x^{\prime \prime}: x \in P\right\} \text { by definition since } \operatorname{Succ}\left(x^{\prime \prime}\right)=\emptyset .
\end{aligned}
$$

It is easy to verify that

$$
B(\widehat{P})=\widehat{B(P)} .
$$

So we may generalize the theorem to

$$
B^{n}\left(S^{n}[P]\right)=\hat{\hat{\hat{P}}}^{n \text { boundings }}
$$

Investigations on the effect of iterated splitting to the dimension [19] lead to the inequality

$$
\operatorname{dim} P \leq \operatorname{dim} S^{n}[P] \leq 2+\operatorname{dim} P \text { for all } n
$$


As a consequence of (7) and (8) we obtain that, for every $n$ there is are partial orders $P$ such that

$$
\operatorname{dim} P-\operatorname{dim} B^{k}(P) \leq 2 \text { for all } k \leq n
$$

(Just take $P=S^{n}[Q]$ for some order $Q$ ). If we choose $Q$, however, to be an $m$ dimensional interval order we obtain a large difference in dimension with the next iteration, i.e.,

$$
\operatorname{dim} P-\operatorname{dim} B^{n+1}(P) \geq m-1 .
$$

\subsection{The interval dimension of subdivisions}

With the next theorem we relate the interval dimension of subdivisions of $P$ to the dimension of $P$. Spinrad [17] showed that the dimension of a subdivision of a partial order can be an arbitrary multiple of its dimension, thus answering Trotter's Problem 4 in [22]. With our result we establish a theoretical framework for his examples.

In this context, partial orders and their diagrams are regarded as directed graphs whose edges $(x, y)$ correspond to ordered pairs and cover pairs $x \prec y$ of $P$, respectively. An edge $(x, y)$ is subdivided by placing a new vertex $z$ in the 'middle' of the edge, i.e., $(x, y)$ is replaced by $(x, z)$ and $(z, y)$. In the case of partial orders we then have to ensure transitivity, i.e., all edges $(a, z)$ with $a \in \operatorname{Pred}[x]$ and $(z, b)$ with $b \in S u c c[y]$ are also added.

The complete diagram subdivision $D S(P)$ is the subdivision of all edges of the diagram of $P$. The complete subdivision $C S(P)$ is the transitive closure of the subdivision of all the edges of $P$. Let $E$ be any set of edges of $P$, we denote the order obtained by subdividing the edges of $E$ with $\operatorname{Sub}(P, E)$. Since $P$ is an induced suborder of each subdivision $\operatorname{Sub}(P, E)$, and since $\operatorname{Sub}(P, E)$ is an induced suborder of $C S(P)$, we obtain

$$
\operatorname{dim}(P) \leq \operatorname{dim} \operatorname{Sub}(P, E) \leq \operatorname{dim} C S(P)
$$

With the next theorem we give an upper bound for $\operatorname{idim} \operatorname{Sub}(P, E)$.

Theorem $4 \operatorname{idim} \operatorname{Sub}(P, E) \leq i \operatorname{dim} D S(P)=i \operatorname{dim} C S(P)=\operatorname{dim}(P)$. 
Proof. Take any embedding of $P$ into $\mathbf{R}^{k}$ with $k=\operatorname{dim}(P)$ and grow the points to obtain an embedding by 'miniboxes'. An interval embedding of a subdivision $\operatorname{Sub}(P, E)$ is then obtained by adding the box with lower extreme corner $u_{x}$ and upper extreme corner $l_{y}$ for the point $z$ subdividing the edge $(x, y) \in E$ - see Figure 2. This gives idim $\operatorname{Sub}(P, E) \leq \operatorname{dim}(P)$, independent of the choice of $E$.
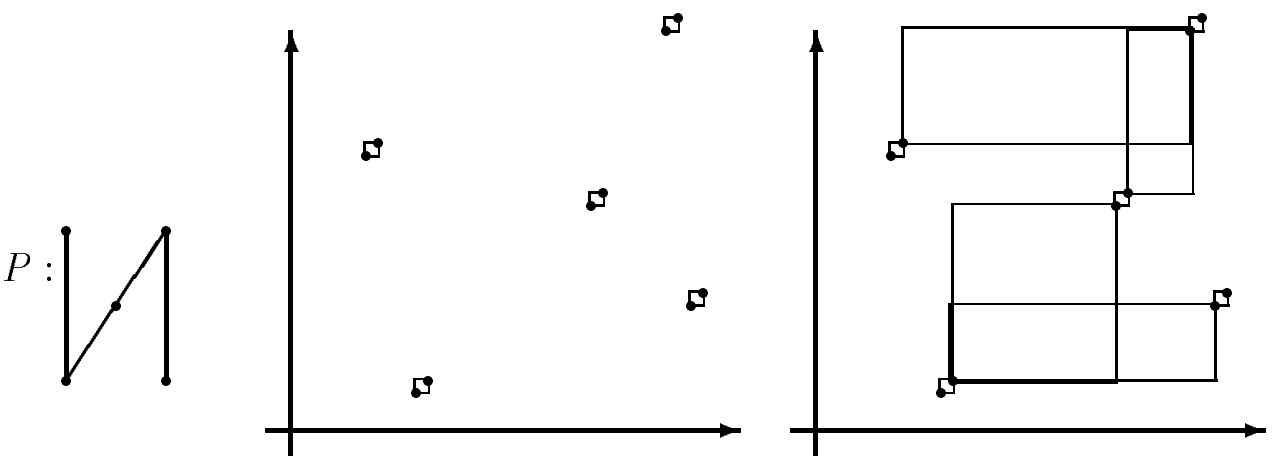

Figure 2: $P$, a minibox embedding of $P$ and the box embedding of $D S(P)$

To prove that $\operatorname{idim} \operatorname{DS}(P)=\operatorname{idim} C S(P)=\operatorname{dim}(P)$ we will show that $P$ can be embedded in $B(D S(P))$. This will give $\operatorname{dim}(P) \leq \operatorname{dim} B(D S(P))$. From Theorem 2 we know idim $D S(P)=\operatorname{dim} B(D S(P))$. Together, we obtain $\operatorname{dim}(P) \leq$ $\operatorname{idim} D S(P)$.

To show that $P$ can be embedded in $B(D S(P))$ we apply the normalizing procedure to the box embedding $\mathcal{I}$ of $D S(P)$ constructed in the first paragraph of the proof. Because of the construction of the box embedding of DS $(P)$, the only changes that occur in normalization are shifts of the left endpoints of intervals corresponding to elements in $\operatorname{Min}(P)$ and the right endpoints of elements in $\operatorname{Max}(P)$. We then embed $P$ into the lower extreme corners of the miniboxes of the normalized representation $\mathcal{I}^{*}$. This gives $\operatorname{dim}(P)=k=\operatorname{idim}\left(\mathcal{I}^{*}\right)=\operatorname{idim}(D S(P))$

Note that we obtained, in fact, a slightly stronger result: If a set $E$ of edges of $P$ contains the edges of the diagram, then $B(S u b(P, E))=V S(P)$, where $V S(P)$ denotes the vertical split of $P$, i.e., the order obtained from $P$ by substituting each vertex by a $2-$ chain. In $[20]$ a distinct proof for $\operatorname{dim}(P)=\operatorname{idim} \operatorname{VS}(P)$ has been given. 


\subsection{Comparability invariance}

For the definition and basic facts on comparability invariance see [10]. Let $\operatorname{Comp}(P)$ be the comparability graph of $P$. We will show that $\operatorname{Comp}(B(P))$ is a comparability invariant of $P$ in the sense that if $\operatorname{Comp}(P)=\operatorname{Comp}(Q)$ then $\operatorname{Comp}(B(P))=\operatorname{Comp}(B(Q))$. Together with Theorem 2 and the known fact that dimension is a comparability invariant, this gives an alternative proof of the comparability invariance of interval dimension in the finite case. The comparability invariance of interval dimension was first shown in [11].

A subset of elements $A$ of $P$ is called autonomous if the relation of elements in $A$ to an element outside $A$ is independent of the element of $A$. More formally, if for any $x \notin A$, whenever $x<a, x>a$ or $x \| a$ holds for some $a \in A$, then it holds for all $a \in A$. If $A$ is an autonomous subset of $P$, then $\operatorname{Pred}(A)$ shall denote the predecessors outside $A$ of any and hence every element of $A$.

Theorem 5 Comp $(B(P))$ is a comparability invariant of $P$.

Proof. Let $A$ be an autonomous subset of $P$. It is enough to show (see, e.g.,[11]), that $\operatorname{Comp}(B(P))=\operatorname{Comp}\left(B\left(P_{A^{d}}^{A}\right)\right)$, where $P_{A^{d}}^{A}$ denotes the order resulting from substituting $A$ by its dual $A^{d}$ in $P$.

Note first that $B(A)=\{L(a), U(a): a \in A\}$ is a closed suborder of $B(P)$. Let $\widehat{B(A)}$ be $B(A)$ without its greatest element $1_{B(A)}$ and its least element $0_{B(A)}$. Our claim is that $\widetilde{B(A)}$ is autonomous in $B(P)$. To see this, observe first that,

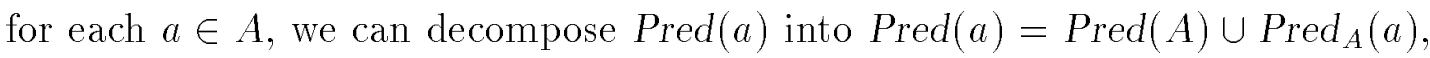
hence, the same is valid for all elements of $\widetilde{B(A)}$. On the other hand, the elements of $B(P) \backslash B(A)$ either contain all of $A$ or their intersection with $A$ is empty. Now if $M \in B(P) \backslash B(A)$ contains all of $A$ then it also contains $\operatorname{Pred}(A)$ and $M$ is above all sets in $\widehat{B(A)}$. If $M \subseteq \operatorname{Pred}(A)$ then $M$ is below all sets in $\widehat{B(A)}$. In all the other cases $M$ is unrelated to all of $\widehat{B(A)}$. This gives the claim.

To settle the theorem we need that $B\left(A^{d}\right)$ and $B(A)^{d}$ are isomorphic orders. To see this, consider a normalized box embedding of $A$ in $\mathbf{R}^{k}$. Its extreme corners are an embedding of $B(A)$ into $\mathbf{R}^{k}$. Flip the embedding, i.e., reverse the relations, this gives an embedding of $A^{d}$ and the extreme corners form an embedding of $B(A)^{d}$. 
As we have seen, autonomous sets in $P$ induce autonomous sets in $B(P)$. The converse, however, is far from being true. Take as $P$ a prime interval order, then $B(P)$ is a chain. Hence $P$ has none but $B(P)$ has $\left(\begin{array}{c}|B(P)| \\ 2\end{array}\right)-1$ nontrivial autonomous sets.

\subsection{Remarks on related results and computational com- pexity}

The transformation $P \rightarrow B(P)$ obviously can be carried out in polynomial time.

The degree of the polynomial of this reduction is not important if we want to decide if $P$ has interval dimension $\geq 3$, since the poset dimension and interval dimension problems have been shown to be $N P$-complete for $k \geq 3$ by Yannakakis [23]. It is, however, a crucial point if we want to decide if $\operatorname{idim}(P) \leq 2$.

Dagan Golumbic and Pinter [4] addressed questions about the comparability invariance of interval dimension and (fast) recognition of interval dimension at most 2. As remarked before, the first problem has been settled affirmatively first in [11]. The recognition problem has been solved independently by several authors. Habib and Möhring [12] use the subposet of $B(P)$ defined by $B^{\prime}(P)=$ $\{L(x): x \in P\}$ together with an algorithm for transitive orientation with side constraints to derive an $O\left(n \cdot n^{a}\right)$ algorithm, where $O\left(n^{a}\right)$ is the best known time for matrix multiplication. Currently $a$ is about 2.37 .

Cheah [3] proposed an algorithm in complexity $O\left(n^{3}\right)$. The same complexity is claimed by Langley [13]. Langley calles the poset $B(P)$ of the present paper the 'predecessor-successor order' of $P$ and shows without the aid of the geometric construction that finding an interval realizer of $P$ is equivalent to finding a linear realizer of $B(P)$.

Spinrad [18] has shown that recognition of 2-dimensional orders only requires $O\left(n^{2}\right)$ operations. Therefore, the bottleneck with the $P \rightarrow B(P)$ approach for the recognition of interval dimension 2 is the computation of the order $B(P)$. With a careful implementation, $B(P)$ can be computed in $O\left(n^{a}\right)$, where again $O\left(n^{a}\right)$ is the best known time for matrix multiplication.

Ma and Spinrad [14] have found an approach which allows to avoid matrix multiplication and leads to a complexity bound of $O\left(n^{2}\right)$. Since this is the best 
known result we outline the ideas behind their algorithm.

First, they construct the open split $S(P)$ of the partial order $P$, for which they want to decide if $\operatorname{idim}(P) \leq 2$. The elements of the open split $S(P)$ are the same as of the split $S[P]$ defined above, the ordered pairs of $S(P)$ are, however, given by the irreflexive relation defining $P$, i.e., $x^{\prime}<y^{\prime \prime}$ in $S(P)$ iff $x<y$ in $P$. They prove that the co-chain covering number of $S(P)$ equals the interval dimension of $P$. A theorem of Yannakakis [23] shows that the co-chain covering number of $S(P)$ and the interval dimension of $S(P)$ coincide. Hence, $\operatorname{idim}(P)=\operatorname{idim}(S(P))$ and we can reduce attention to the recognition of interval dimension 2 for bipartite orders, i.e., to orders of height one.

The transformation of the interval dimension 2 problem for bipartite orders to the dimension 2 problem is done in two steps. In the first step it is checkued whether the bipartite order $Q$ has a chordal bipartite comparability graph. A chordal bipartite graph is a bipartite graph without any induced cycle $C_{n}$, $n \geq 6$. If $\operatorname{Comp}(Q)$ is not chordal bipartite, then $Q$ has to contain a crown and, therefore, $\operatorname{idim}(Q) \geq 3$. Otherwise, the second step is started. In this step $Q$ is transformed into an order $P_{Q}$ which can be obtained by contracting autonomous chains in $\operatorname{Stack}(Q)$ and, hence, has the same dimension as $\operatorname{Stack}(Q)$. The stack operation has been introduced by Trotter [21]. He proves that for bipartite posets $\operatorname{dim} \operatorname{Stack}(Q)=i \operatorname{dim}(Q)$. As the construction of $B(Q)$, the construction of $P_{Q}$ and $\operatorname{Stack}(Q)$ requires information about the containment relation of neighborhoods in $Q$. For chordal bipartite graphs this information can be computed in $O\left(n^{2}\right)$ using a technique called doubly lexical ordering. Since after passing the first step we know that $Q$ is chordal bipartite, we can construct $P_{Q}$ and apply Spinrad's dimension 2 algorithm, all in $O\left(n^{2}\right)$.

\section{Acknowledgement}

We are greatful to an anonymous referee for his fruitful suggestions, which improved the presentation of the paper.

\section{References}

[1] K.B. Bogart, I. Rabinovich and W.T. Trotter, A Bound on the Dimen- 
sion of Interval Orders, Journal of Comb. Theory (A) 21 (1976), 319-328.

[2] O. Cogis, On the Ferrers Dimension of a Digraph, Discrete Math. 38 (1982) $47-52$.

[3] F. Cheah, A Recognition Algorithm for Il-graph, Toronto Univ., Department of Computer Science, Technical Report 246/1990 .

[4] I. Dagan, M.C. Golumbic and R.Y. Pinter, Trapezoid Graphs and their Coloring, Discrete Applied Math. 21 (1988) 35-46.

[5] B. Dushnik and E. Miller, Partially Ordered Sets, Amer. J. Math. 63 (1941) 600-610.

[6] S. Felsner, Orthogonal Structures in Directed Graphs, Journal of Comb. Theory (B), to appear.

[7] P.C. Fishinun, Interval Orders and Interval Graphs, Wiley, New-York, 1985.

[8] A. Frank, On chain and antichain families of a partially ordered set, Journal of Comb. Theory (B) 29 (1980), 176-184.

[9] Z. Füredi, V. RödL, P. Hajnal and W.T. Trotter, Interval Orders and Shift Graphs, Preprint 1991.

[10] M. Haвiв, Comparability invariants. Annals Discrete Math., 23 (1984) 331386 .

[11] M. Habib, D. Kelly and R.H. Möhring, Interval dimension is a comparability invariant. Discrete Math., 88 (1991) 211-229.

[12] M. Habib AND R.H. Möhring, Recognition of partial orders with interval dimension two via transitive orientation with side constraints, Preprint 244 TU-Berlin 1990.

[13] L.J. Langley, A Recognition Algorithm for Orders of Interval Dimension Two, Preprint Dartmouth College 1991.

[14] T. Ma And J. SPinrad, An $O\left(n^{2}\right)$ Time Algorithm for the 2-Chain Cover Problem and Related Problems, Proc. second annual acm-siam symp. on discr. alg. (1991) 363-372.

[15] O. Ore, Theory of Graphs, Amer. Math. Soc. Colloq. Publ. Vol. 38 (1962).

[16] I. Rabinovich, A Note on the Dimension of Interval Orders, Journal of Comb. Theory (A)25 (1972), 68-71.

[17] J. Spinrad, Edge Subdivision and Dimension, Order 5 (1989) 143-147.

[18] J. Spinrad, On comparability and permutation graphs, SIAM J. Comput 14 (1985) 658-670.

[19] W.T. Trotter and J.I. Moore, The dimension of planar posets, Journal of Comb. Theory (B) 22 (1977) 54-67. 
[20] W.T. Trotter and J.I. Moore, Characterization Problems for Graphs, Partially Ordered Sets, Lattices and Families of Sets, Discrete Math. 16 (1976) 361-381.

[21] W.T. Trotter, Stacks and Splits of Partially Ordered Sets, Discrete Math. 35 (1981) 229-256.

[22] W.T. Trotter, Problems and Conjectures in Combinatorial Theory of Ordered Sets, Annals of Discrete Math. 41 (1989) 401-416.

[23] M. Yannakakis, The Complexity of the Partial Order Dimension Problem, SIAM J. Alg. Disc. Meth. 3 (1982) 351-381. 